Faraday Discuss. Chem. Soc., 1983, 75, 103-115

\title{
On the Theory of Intramolecular Energy Transfer
}

\author{
By Rudolph A. Marcus \\ Arthur Amos Noyes Laboratory of Chemical Physics, California Institute of \\ Technology, Pasadena, California 91125, U.S.A.
}

Received 21st December, 1982

We consider the distinguishing features of two main types of classical anharmonic motion in molecules, their quantum parallels, and conditions that classical chaos also be sufficient for "quantum chaos". Implications are considered for experimental reaction rates, R.R.K.M. theory, spectra and a possible type of system for intramolecular laser-selective chemistry. A theory of intramolecular energy transfer between two ligands of a heavy atom is described for a system which may contain many coordinates. It is partly statistical and, for the modes of each ligand which communicate through the heavy atom, dynamical.

\section{INTRODUCTION}

Quasiperiodic and chaotic motion in molecules, and more generally in anharmonic systems, has been the subject of considerable interest in recent years, and of many conferences and reviews [see e.g. ref. (1)-(14)]. From a chemist's viewpoint several main questions are the following: (1) What are the main distinguishing features of the different types of classical mechanical anharmonic motion (quasiperiodic versus chaotic)? (2) What is known about the corresponding quantum-mechanical behaviour? Does classical "chaos" imply a quantum " chaos"? How might one define the latter? (3) Do quasiperiodic and chaotic behaviour always differ in their collision-free intramolecular energy redistribution, or is some other aspect of the anharmonic motion equally or more important? (4) What implications do the theoretical anharmonic studies have for experimental data, e.g. for the rates of unimolecular processes, for spectra and for intramolecular laser-selective chemistry? How would one differentiate experimentally between the various types of behaviour, taking state preparation into account? (5) What does statistical (e.g. R.R.K.M.) behaviour imply in terms of the underlying dynamics?

We address these questions in sections $2-5$ from a unified viewpoint. We consider a possible type of system for intramolecular laser-selective chemistry (section 6) and present a theory of intramolecular energy transfer between two parts of a molecule joined by a heavy atom (section 7). A criterion for overlapping avoided crossings, and conditions that classical chaos imply " quantum chaos" are given in section 3. Some of the material is presented here to set the background on some points about which there has been some confusion or misunderstanding in the literature. We have recently reviewed the literature on quasiperiodic versus chaotic motion. ${ }^{1}$ Extensive references are given there and we shall sometimes refer to that and other reviews instead of to the original articles. 


\section{CLASSICAL QUASIPERIODIC VERSUS CHAOTIC MOTION ${ }^{1-14}$}

Quasiperiodic motion implies classically that for a system of $N$ coordinates the system moves on an $\mathrm{N}$-dimensional surface in $2 \mathrm{~N}$-dimensional phase space (or less than $N$ dimensions if the motion is degenerate). The surface (a torus) is determined by $N$ constants of the -motion (the action variables) and the trajectory uniformly covers that surface. The time dependence of any coordinate can be expressed as a Fourier series in the $N$ phases (the angle variables) and thereby in terms of $N$ fundamental frequencies (less if degenerate), plus combinations and overtones. The power spectrum of any dynamical variable can be obtained by computing its autocorrelation function using a classical trajectory $;^{1}$ the above frequencies appear as sharp lines.

Mathematically, classical quasiperiodic motion for a system of $N$ coordinates exists if there are $N$ integrals of the motion whose Poisson brackets with each other vanish. If this applies to all initial conditions the system is " integrable." In general, most dynamical systems are not integrable, but for a large fraction of the initial conditions at small perturbations from an integrable system these $N$ commuting integrals of the motion do exist, according to the KAM (Kolmogorov, Arnold, Moser) theorem. The resulting quasiperiodic motion for such an initial condition then exists stably for infinite time for these initial conditions.

In a classical quasiperiodic trajectory there can, in the absence of internal resonances, be very unequal distributions of energy among the various modes of vibration for infinite time, a point to which we shall return later. Internal resonances (particularly, but not restricted to, $1: 1$ resonances) cause extensive periodic sharing of the energy among the participating zeroth-order (harmonic) modes. Isolated resonances provide another example of quasiperiodic motion and have been so treated in the literature.

Usually, as the energy of the system increases, or as the perturbation from an integrable system (one with $N$ actions) increases, an increasing fraction of the trajectories becomes chaotic. In classically chaotic motion all of the $N$ action variables no longer exist, although some may, and correspondingly instead of having a sharp-lined power spectrum the spectrum becomes " grassy". (It may become "broadened", but there are not enough cycles of the trajectory to make it a continuum.) Each trajectory becomes very sensitive to its initial condition, and initially neighbouring trajectories separate in phase space exponentially with time. In contrast, neighbouring quasiperiodic trajectories separate linearly in time.

Nevertheless, chaotic behaviour of an individual trajectory need not be the same as microcanonically averaged behaviour. In a two-oscillator system, in particular, it is confined between adjacent tori which have persisted.

Chirikov's approach to the onset of chaotic motion is the following, and involves an "overlap of resonances". 7 A particular trajectory for some initial condition (specific actions) has a commensurable set of frequencies (a resonance) and nearby tori have almost commensurable frequencies near this ratio, and are within the "width of this resonance " in action space: two or more of the independent angle variables (phases) in these trajectories can become correlated. Nearby there is another periodic trajectory with a different commensurability and so the centre of a different resonance. Around it are tori with near-commensurability almost equal to this new commensurability and this resonance has its own " resonance width". Although each resonance is not in itself chaotic (one can define actions for any trajectory in this width), an overlap of these widths in phase space initiates the onset of chaos, according to Chirikov, a chaos which under stronger perturbations or higher energies becomes increasingly widespread. 
In addition to quasiperiodic ${ }^{1}$ and chaotic ${ }^{14}$ motion another type of motion has been found recently in hard-walled " rational billiards" problems. ${ }^{15}$ The system is unusual in that it lies on a special two-dimensional surface in four-dimensional phase space, termed a "multiply handled sphere," which is not a torus. The action integrals do not exist, and the surface has been termed pseudointegrable. ${ }^{14,15}$ It is apparently not known whether any such surface applies to any molecular system. Initially neighbouring trajectories would presumably separate rapidly when one proceeded along a handle and the other, on the other side of a bifurcation, along the main sphere. Such "bifurcations" would presumably cause irregularities in the classical power spectrum of a trajectory on that surface. Thus this special case has some properties of a chaotic system (even " overlapping avoided crossings"), ${ }^{15}$ although the trajectory lies on a surface of the same dimensionality as a torus. We omit consideration of this special case.

\section{QUANTUM BEHAVIOUR}

The semiclassical quantum analogue of quasiperiodic motion is now well understood. [It is reviewed in ref. (1), (6) and (11).] As long as action variables exist for the trajectory, one can introduce an Einstein-Brillouin-Keller semiclassical quantization of them (a WKB approximation for solving the Schrödinger equation). Keller showed how to do this for non-separable systems with hard-walled potentials ${ }^{16}$ and more recently Eastes ${ }^{17}$ and Noid ${ }^{18}$ showed how to determine action integrals from quasiperiodic classical trajectories from non-separable systems with smoothly varying potentials. Previously, the only such systems successfully treated had been those in which one can separate variables or in which one can apply classical canonical perturbation theory to obtain the actions. A variety of perturbative or perturbativeiterative methods have since been presented. [For reviews see ref. (1), (6) and (11).]

When trajectory or perturbative methods are applied in their simplest form, one has a "primitive" semiclassical quantization. This quantization is satisfactory in most instances when the motion is quasiperiodic. Systems with and without internal resonances have now been quantized in this way. However, when the quantized trajectory occurs near a separatrix, i.e. near a surface which separates tori describing two different types of motion, a uniform semiclassical quantization is needed. Examples include the one-dimensional double-well problem, ${ }^{19}$ the pendulum problem, the Hénon-Heiles system (the border between librating and precessing trajectories), ${ }^{20}$ local-mode-normal-mode description of triatomic molecules ABA, ${ }^{21}$ group local-mode treatment of polyatomic molecules $\mathrm{ABC}$ in which $\mathrm{A}$ and $\mathrm{C}$ are each a set of atoms and $B$ is heavy, ${ }^{22}$ and avoided crossings (" anti-crossings ") in quantum mechanics. ${ }^{23}$ All of these problems are either or have been approximately reduced to that of one dimension.

Some of the properties of the classical quasiperiodic trajectories have parallels in those of the corresponding quantum-mechanical states: ${ }^{1}$ the spectrum, which was relatively simple for the classical quasiperiodic system, is comparatively simple in the quantum-mechanical system. (Of course, when there are a number of coordinates there can be many regular sequences of overtones and combinations. Unravelling the spectrum may then become impractical.) One difference from a classical mechanical spectrum is that the latter is obtained from a trajectory at some given energy, and so the classical overtones are exact multiples of the fundamentals at that energy. In the quantum case each spectral line involves a transition between two energies, and so is at best only centred on some energy. Since this mean energy increases with each overtone, the exact multiple relation is lost. Nevertheless there is a regular progression 
of spectral lines (overtones and combinations) with gradually changing spacing. Taking this effect into account in the comparison between classical and quantum spectra, so that the individual spectral lines are always compared at a mean action corresponding to the mean quantum number, there was good agreement not only of the positions of the spectral lines but also of their intensities for the cases studied in ref. (24).

There is also a correspondence of the "shape" of the wavefunction and the corresponding semiclassical trajectory (i.e. the one whose actions match the quantum numbers via a semiclassical relation). ${ }^{1}$ They display regular (rather than chaotic) contour patterns. Indeed, semiclassically calculated wavefunctions are expected to compare well with the quantum-mechanical ones, if any needed uniform approximation is introduced. Correspondingly, there is also a correspondence of the so-called Poincare surfaces of section with Wigner distribution functions (calculated in a particular way). ${ }^{25}$ One thus expects that, apart from tunnelling and interference contributions to any particular problem, there will be a close correspondence between various reduced properties calculated by averaging using a wavefunction or by phase averaging over the corresponding classical torus.

Quantum-mechanically an integrable system could be defined, by analogy with the classical definition ${ }^{10}$ of commuting Poisson brackets, as a system for which the operators for the $N$ integrals of the motion of an $N$-coordinate system commute with each other. A consequence of this definition is that there are sequences of quantum numbers $\left(n_{1}, \cdots, n_{N}\right)$, just as there are individual actions $\left(J_{1}, \cdots, J_{N}\right)$, which describe the state of the system, rather than just one quantum number ordering the states. Correspondingly there are expected to be "regular patterns" in the wavefunctions and regular progressions of spectral lines. It is quite likely, by analogy with the KAM theorem, that this behaviour continues to be true (at least approximately now) for small perturbations from an integrable system. However, only in the limit $\hbar=0$ may it be exactly the case.

In summary there is a close relation between quantum states and the related quasiperiodic trajectories, when the latter exist. The former have "regular wave patterns" and " regular spectra."

Since quasiperiodic motion for the general case, i.e. the case which is not integrable, has not been precisely defined for quantum systems, but only by semiclassical correspondence, it is not surprising that no generally accepted definition of quantum chaos exists. By analogy with the corresponding classical case one could compare two wavepackets initially neighbouring in some phase-space sense and examine the nature of their separation in time, exponential or linear, of values of some phase space operator in the two packets. In contrast to classical mechanics, however, the two packets would no longer each be at specified energies, and the results might depend on their width and on the states chosen for the packet, there being at least several quantum states rather than just one. A number of investigators, including Heller, Brumer and Shapiro, Weissman and Jortner, and Pechukas, have discussed the evaluation of wavepackets from various viewpoints [partly reviewed in ref. (1)].

Another criterion for distinguishing which quantum states are analogous to quasiperiodic and chaotic trajectories would be the spectrum--whether or not irregularities exist. ${ }^{11}$ Recently we described a way that irregularities can occur [ref. (1) and references cited therein]. States can mix wave patterns via avoided crossings of eigenvalues. Avoided crossings belonging to a certain sequence would show a regular progression of the spacings. (A single classical resonance can embrace within its width many, few, or no pairs of states which show avoided crossings.) Like an isolated classical resonance, ${ }^{7}$ an avoided crossing would not in itself constitute chaos. ${ }^{1}$ 
We next consider overlapping avoided crossings. A criterion for this overlap is obtained below.

Each avoided crossing has some "width" over which the two wavefunctions are changing rapidly in the plots of the two eigenvalues versus a perturbation parameter $\lambda$. One can define a "width" for an avoided crossing in the following way. The twostate pair of eigenvalues are, using degenerate perturbation theory, given by

$$
E_{ \pm}=\frac{1}{2}\left\{H_{11}+H_{22} \pm \sqrt{ }\left[\left(H_{11}-H_{22}\right)^{2}+4\left|H_{12}\right|^{2}\right]\right\}
$$

where $H_{11}$ and $H_{22}$ depend on $\lambda$ and where, for the purposes of the present illustration only, $H_{12}$ is constant. The difference of the slopes of the two " crossing" eigenvalue plots in the vicinity of the avoided crossing (i.e. at a value of $\lambda$ where $H_{11}=H_{22}$ ) is denoted by $s$ :

$$
s=\partial\left(H_{11}-H_{22}\right) / \partial \lambda \quad \text { at } H_{11}=H_{22} \text {. }
$$

The " half-width" $w$, in $\lambda$-space, of the avoided crossing is the distance in $\lambda$, from the crossing-point, needed for $4\left|H_{12}\right|^{2}$ to be comparable to $\left(H_{11}-H_{22}\right)^{2}$. Hence

$$
w \approx 2\left|H_{12}\right| / s .
$$

$2\left|H_{12}\right|$ is the splitting of the two states at the $\lambda$ where $H_{11}=H_{22}$. With the half-width defined as in eqn (3.3) one sees that overlap of two avoided crossings A and B occurs when the distance between their respective centres $\Delta \lambda$ satisfies

$$
\Delta \lambda<w_{\mathrm{a}}+w_{\mathrm{b}}
$$

and when they simultaneously have a state in common. One can extend the arguments to a multi-parameter space. The avoiding curves become a special case of the well known conical intersection. ${ }^{10}$

One view of "quantum chaos" 1,14 is that it corresponds not to an isolated avoided crossing but to overlapping avoided crossings. The latter would yield irregularities in the spectrum and a complex contour pattern for the wavefunction of a quantum state, reflecting the involvement in several regularly patterned wavefunctions in this state. ${ }^{1}$ The generation of statistical (" scrambled ") wavefunctions by overlapping avoided crossings, ${ }^{26}$ and the properties of the former, have both been discussed recently. ${ }^{27}$

"Quantum chaos" does not ${ }^{28-30}$ automatically follow when the corresponding classical trajectories are chaotic. If, for the particular actions of interest, the width of the classical resonance is less than $h$, it has little consequence quantummechanically. (More precisely, the splitting at the avoided crossing becomes very small. $^{23}$ If it is much larger than $h$ this classical resonance can support a number of the pairwise avoided crossings, each arising in a sequence of eigenvalues. If the overlap of two classical resonance widths is so small that it does not contain even a single quantum state, this overlap of the classical resonances is of little consequence quantum-mechanically. If there is a quantum state in this classical overlap width, it participates in the two overlapping classical resonances and hence in both sets of avoided crossings, and eqn (3.4) is fulfilled. That is, it participates in overlapping avoided crossings.

The conditions that classical chaos (assumed to involve overlapping resonances) also yields quantum chaos (assumed to involve overlapping avoided crossings) are that (i) the width of a Chirikov resonance exceeds $h$, i.e. that it contains at least one quantum state, ${ }^{28-30}$ (ii) that the overlap width of the two overlapping resonances also contains at least one quantum state, ${ }^{28}$ and (iii) that the centre of the resonance be close to a quantum state. ${ }^{28,29}$ 
The half-width of the resonance is, in the notation of section $6,2 \sqrt{ }(A / \Omega)$, where $A$ and $\Omega$ are properties of the resonant Hamiltonian and where the width of the angle space is $2 \pi$ (using the action-angle variables introduced in section 6). Thus the first condition for " quantum chaos" is *

$$
[4 \sqrt{ }(A / \Omega)] \hbar>1 \text {. }
$$

The second condition can be written as follows: If $\Delta J_{i j}$ is any projection of the overlapping resonance zone on any two-dimensional action space $\left(J_{i} J_{j}\right)$ then we require that there be at least one system of orthogonal axes in action space such that $\dagger$

$$
\Delta J_{i, j} / \hbar>1, \text { for all } i, j \text { pairs. }
$$

[See also ref. (9) and (30).] One system which has been extensively studied is the 99-state Hénon-Heiles system, an anharmonic coupled oscillator system with $C_{3 v}$ symmetry and nearly $1: 1$ resonant. It becomes classically chaotic in part at the higher energies. In contrast, it has regular sequences of eigenvalue with slowly varying spacings, ${ }^{31}$ its wavefunctions show regular patterns of their contour plots, ${ }^{32}$ and its eigenvalues are semiclassically very well approximated by those of an integrable Hamiltonian. ${ }^{20,33}$ (This second Hamiltonian is a perturbation approximation to the former, and so has well defined action variables.) Incidentally most semiclassical quasiperiodic states in this system show extensive redistribution of energy; they correspond to precessing trajectories. ${ }^{34}$ We conclude that the 99 -state system is quantum-mechanically " regular".

For this same system the Poincaré surface of section of a particular chaotic trajectory resembled in coverage the Wigner distribution function of a quantum state at the same energy. ${ }^{25}$ However, one cannot conclude that the latter quantum state is chaotic. Because there are only two coordinates the same chaotic trajectory is trapped between two residual tori, each of which would also be expected to have Poincaré surface of sections of rather similar shape to the Wigner distribution function. We are currently investigating this possibility.

\section{INTRAMOLECULAR ENERGY REDISTRIBUTION}

We have already noted that one can have extensive classical-mechanical intramolecular energy redistribution even in a quasiperiodic system, when there is an internal resonance, but that the redistribution is quasiperiodic rather than chaotic. The precessing trajectories in the Hénon-Heiles system provide an example. Similarly, in quantum systems where there is a low order (e.g. $1: 1,2: 1$, etc.) degeneracy or near-degeneracy (as in Fermi resonance) there is an extensive energy sharing ${ }^{35}$ or, if one introduces a suitable wavepacket, energy redistribution among zeroth-order modes.

Another example of equipartitioning of energy among zeroth-order (harmonic) modes, when one such mode was excited, was for an integrable system, the Toda lattice. Six-atom and fifteen-atom systems were investigated. ${ }^{36,37}$ The vibration frequencies were reported for the six-atom system ${ }^{37}$ and several were commensurable. Thus internal resonances, rather than quasiperiodic versus chaotic motion per se, played a major role in the equipartitioning in that system, as well as in the precessing trajectories of the Hénon-Heiles system.

This importance of internal resonances for energy redistribution is also seen in a study of a seven-atom system $\mathrm{C}-\mathrm{C}-\mathrm{C}-\mathrm{M}-\mathrm{C}-\mathrm{C}-\mathrm{C}$, where $\mathrm{M}$ is a heavy atom and

\footnotetext{
* Eqn (12) of ref. (28) actually gives the half-width and should be multiplied by a factor of 2 .
}

$\dagger$ Eqn (3.6) replaces eqn (14) of ref. (28), which is correct only for a two-coordinate system. 
the oscillators are Morse oscillators. ${ }^{38}$ When the energy of excitation of one of the bonds is fairly high ( 0.3 of the bond dissociation energy) there is relatively little transfer of energy from the first half of the molecule to the second, although there is extensive energy transfer among the bonds of the first half themselves. When the excitation energy is instead small, only 0.05 of the bond dissociation energy, there is complete transfer. Finally, when the Morse oscillators are replaced by harmonic ones, with 0.3 of the Morse bond dissociation energy, there is again complete transfer. We have since determined the spectrum of each trajectory. The motion in all the above cases is quasiperiodic.

The above effects can be explained in terms of internal resonances. When the energy of the first part of the molecule is high, its frequencies are red-shifted from those of the second part and there is comparatively little energy transfer. Because of the quasiperiodicity the observed non-equilibrating behaviour should persist classically for infinite time. Transfer is complete in the other two cases because of resonance. It is essential for the effect that the two parts of the molecule be coupled by a small perturbation. For example, when the mass of $\mathrm{M}$ in $\mathrm{C}-\mathrm{C}-\mathrm{C}-\mathrm{M}-\mathrm{C}-\mathrm{C}-\mathrm{C}$ is reduced by a factor of two there is now extensive energy-sharing at the excitation energy of 0.3 dissociation energy. ${ }^{38}$ (The effect of $\mathrm{C}-\mathrm{M}-\mathrm{C}$ bond angle was also studied; it changes the effective mass of M.) A close analogy is the coupled twopendulum problem. When the frequencies are not commensurate there is not complete energy transfer. Theoretical studies of resonant effects in triatomic molecules have been described recently. ${ }^{21,39-41}$

We have noted that in both the quasiperiodic and chaotic cases there can be extensive intramolecular energy transfer in the presence of internal resonances. (In the quantum case, the collisional or optical excitation is a " pulse" and generates a wavepacket which then shows little or extensive redistribution, depending on the nature and energies of the states it embraces.) However, although the redistributed energy may be equipartitioned, a single resonance is not " microcanonical " and so R.R.K.M. theory would not be automatically satisfied by it. This point is easily established by an example of a $1: 1$ resonance.*

Incidentally, classically the vibration phases of the isolated-molecule phase average themselves in the non-degenerate case. In the case where any zeroth-order degenerate vibrations are anharmonically coupled, they undergo a slow phase averaging also. There is no need for collisions to cause this phase averaging. Although isolated resonances do not create a microcanonical distribution even among participating modes, the resonant coupling of the resonant modes with other modes could yield a more microcanonical distribution. (The $J_{\varphi}{ }^{2}$ in the preceding footnote would no longer be approximately constant.)

Thus R.R.K.M. behaviour is consistent with rather " chaotic" quantum states. What is not clear as yet is how chaotic the system has to be. Particularly interesting is that highly specific excitation, namely of $\mathrm{CH}$ overtones in molecules, may have produced largely statistical results [cf. discussion in ref. (1)], but very few systems have been studied thus far.

Excitation of molecules typically involves a " pulse "- such as an optical pulse or a collision. The pulse will typically involve the excitation of a wavepacket, a group of states. After some initial dephasing period the system will then behave as an ensemble of systems, each disappearing according to its own rate law, and not be a single

* One can define ${ }^{34}$ an internal angular momentum $J_{\varphi}$. At large values of $J_{\varphi}, J_{\varphi}{ }^{2}$ is roughly constant. The conjugate phase $\varphi$ is (slowly) varying and so phase-averages itself. There is a lower limit to the action $J_{x}$ of the $x$ mode, because $J_{\varphi}{ }^{2} \neq 0$. Thus, $J_{x}$ and $J_{y}$ are not microcanonically distributed. The actual distribution function is omitted here for brevity. 
exponential (" intermediate-level structure"), if the exact eigenfunctions are not "scrambled." However, when the density of states coupled to the excited initial "state" is so high as to become essentially a continuum, one obtains a single exponential decay, at least with the usual assumptions of radiationless transition theory, provided the pulse width is sufficiently large to embrace all zeroth-order coupled states [e.g. ref. (42) and references cited therein].

\section{MOLECULAR SPECTRA}

The spectrum will depend on the nature of the underlying dynamical motion. When that motion is the analogue of quasiperiodic, or if the wavefunctions are not too "scrambled" (i.e. if a relevant state does not participate in many overlapping avoided crossings) one expects regular sequences of spectral lines. (Even in the classical chaotic case, however, there is expected to be some propensity for the positions of the overtones.)

If wavefunctions were considerably scrambled Franck-Condon factors in a vibronic excitation would yield an appropriate " dispersed " absorption of fluorescence band: many states of neighbouring energies would have comparable Franck-Condon factors. The sharpness of the low-lying excitation spectral lines corresponding to low vibrational excitation in aromatics, and of the fluorescence, indicates little scrambling. However, at higher energies the dispersed fluorescence in Parmenter's, ${ }^{43}$ Levy's ${ }^{44}$ and in Smalley's ${ }^{45}$ work suggests some scrambling.

\section{INTRAMOLECULAR LASER-SELECTIVE CHEMISTRY}

Some evidence that the intramolecular energy transfer between two ligands attached to a heavy atom may be slow has been given by Rogers et al., based on an interpretation of data of the chemical activation reaction of $\mathrm{F}$ with $\mathrm{Sn}(\text { allyl })_{4}{ }^{46}$ The theoretical dynamics of energy interchange between two ligands in $\mathrm{C}-\mathrm{C}-\mathrm{C}-$ $\mathrm{Sn}-\mathrm{C}-\mathrm{C}-\mathrm{C}$ were considered recently ${ }^{38}$ These various results suggest the possibility of intramolecular laser-selective chemistry: one chooses a molecule in which one reaction can occur in the vibrationally-excited ligand and a distinguishable kind of reaction in the other ligand(s). The excitation could be induced by $\mathrm{CH}$ overtone excitation, infrared multiphoton absorption, or chemical activation. From the results we obtained earlier, ${ }^{38}$ the kinetic-energy coupling between the two ligands would be less the larger the mass of the metal atom and the more the ligand-metalligand bond angle differs from $180^{\circ}$. Other things being equal, the greater the excess energy per bond (in the quasiperiodic regime at least) the more the frequencies of the excited part of the molecule are anharmonically red-shifted. Thus if that part of the molecule and the remaining ligands are in resonance when each has zero energy, they should become off-resonant with increasing excitation energy, and there should be less energy transfer or (quantum-mechanically) a reduced rate of transfer.

The calculations we have described thus far have been in the quasiperiodic regime. The onset of chaos would have several effects, one being to broaden the vibrational spectrum of the excited ligand and increase the possibility of some resonance. However, further excitation in the chaotic regime would further red-shift the frequencies in the excited part of the molecule and so would decrease the resonance (if they were in resonance at low energy). Some calculations in this chaotic regime are being attempted, although numerical errors are large in this regime.

Since the excess energy per bond is an important factor in the effect, and since it is not practical to make detailed calculations, it would be useful to combine a statistical 
theory with dynamical calculations so as to treat larger systems. Such a type of theory is described in the next section.

\section{THEORY OF INTRAMOLECULAR ENERGY TRANSFER ACROSS A HEAVY ATOM}

We consider that one ligand is excited by any one of several sources, such as those described in section 6. We divide the excited ligand into two parts, one of which (mode 1) is capable of promoting intramolecular resonant energy transfer to another ligand of the heavy atom M. The case where there may be more than one such mode is included later. The excitation energy is assumed to rapidly redistribute itself within the excited ligand, and so a statistical approximation will be used to calculate the chance that mode 1 has an approximate action $J_{1}$. ( $J_{1}$ here denotes the conventional action divided by $2 \pi$. The angle conjugate to it has a range $0-2 \pi$.)

To apply the resonance theory described by eqn (7.3)-(7.12) below it is necessary to know what value to use for $J_{1}$ and, if the second ligand is at least thermally excited, what to use for $J_{2}$. We do this first for $J_{1}$, and an analogous argument would be used for $J_{2}$.

We first calculate the distribution function $P\left(J_{1}\right)$ for $J_{1}$. We consider the excited ligand as having an energy in the range $(E, E+\mathrm{d} E)$, and the approximate energy of mode 1 as being $E_{J_{1}}$. Assuming a microcanonical distribution and counting quantum states we have

$$
P\left(J_{1}\right) \mathrm{d} J_{1}=\rho^{\prime}\left(E-E_{J_{1}}\right) \mathrm{d} J_{1} / \rho(E) \hbar
$$

where $\rho$ is the density of quantum states of the excited ligand, $\rho^{\prime}$ is that for the part of the ligand not including mode 1 , and the phase-space volume element for a given $\mathrm{d} J_{1}$ is $2 \pi \mathrm{d} J_{1}$. It, divided by $h$, is the number of quantum states which it contains, i.e. $\mathrm{d} J_{1} / \hbar$.

The mean of $J_{1}, \tilde{J}_{1}$, in this isolated ligand and the mean-square deviation are, of course, $\int J_{1} P\left(J_{1}\right) \mathrm{d} J_{1}$ and $\int\left(J_{1}-\bar{J}_{1}\right)^{2} P_{1} \mathrm{~d} J_{1}$, integrated over all energetically allowed $J_{1}$. If classical values are used for $\rho^{\prime}$ and $\rho$, the $\hbar$ cancels.

We use as a value for $J_{1}$ some value in the above range, $\bar{J}_{1} \pm \sqrt{ }\left[\left(\Delta J_{1}\right)^{2}\right]$, e.g. $\bar{J}_{1}$, but we recognize that there can be fluctuations in $J_{1}$ and in a more complete calculation will include them. If the modes 1 and 2 are off-resonance for $J_{1}=\bar{J}_{1}$ they may be closer to resonance for some $J_{1}$ in the above range. The value of $\bar{J}_{1}$, the resonant centre $J_{1}$ r the resonant width (defined below) and the above range of $J_{1}$ values defined above all become important factors in the energy transfer.

We consider energy transfer between mode 1 , of action $J_{1}$, to an accepting mode in another ligand of $\mathrm{M}$, mode 2 with action $J_{2} . \quad J_{2}$ and $J_{1}$ are both conventional actions divided by $2 \pi$. Extensive energy transfer can occur if $J_{1}$ and $J_{2}$ fall within the " width" of an intramolecular resonance. To find the conditions for such a transfer we adapt the standard treatment ${ }^{7}$ of an isolated non-linear resonance. The Hamiltonian for modes 1 and 2 and for the contribution of $M$ is written as a sum

$$
H \cong H_{1}\left(J_{1}\right)+H_{2}\left(J_{2}\right)+H_{\mathrm{M}}
$$

where $H_{\mathrm{M}}$ contains, via kinetic- and possibly potential-energy coupling, momenta and/ or coordinates of modes 1 and 2. $H_{\mathrm{M}}$ is next expanded in a Fourier series of these angle variables for those modes. The general term contains cosine and/or sine functions of $m_{1} \varphi_{1}+m_{2} \varphi_{2}$, where $m_{1}$ and $m_{2}$ are integers and $\varphi_{1}$ and $\varphi_{2}$ are angle variables conjugate to $J_{1}$ and $J_{2}$. 
There is a resonance when the angular frequencies $\omega_{i}=\partial H_{i} / \partial J_{i}$ satisfy a relation

$$
n_{1} \omega_{1}\left(J_{1} \mathrm{r}\right)-n_{2} \omega_{2}\left(J_{2}^{\mathrm{r}}\right)=0
$$

at some value $\left(J_{1}^{\mathrm{r}}, J_{2}^{\mathrm{r}}\right)$ of $\left(J_{1}, J_{2}\right)$. This value is the " resonance centre." $n_{1}$ and $n_{2}$ are positive integers. The angle $n_{1} \varphi_{1}-n_{2} \varphi_{2}$, whose time derivative is approximately the frequency $n_{1} \omega_{1}\left(J_{1}\right)-n_{2} \omega_{2}\left(J_{2}\right)$, is slowly varying when $J_{1}$ and $J_{2}$ are sufficiently in the neighbourhood of $\left(J_{1}^{\mathrm{r}}, J_{2}^{\mathrm{r}}\right)$.

We consider the case where there is a resonant term in the expansion of $H_{\mathrm{M}}$ in the double Fourier series in $\varphi_{1}$ and $\varphi_{2}$, i.e. a term such as $\cos \left(n_{1} \varphi_{1}-n_{2} \varphi_{2}\right)$, which we denote by $\cos \alpha$. Coupling of modes 1 and 2 also occurs if there is $\cos 2 \alpha$, sine terms, etc., but for simplicity of presentation we consider this $\cos \alpha$ term only. Its coefficient will be a function of $J_{1}$ and $J_{2}$, which we replace as usual by their values at the centre of the resonance, $J_{1}{ }^{\mathrm{r}}$ and $J_{2}{ }^{\mathrm{r}}$.

To treat the dynamical problem we proceed in a standard way, and introduce a transformation into new action-angle variables $\left(J_{\alpha}, \alpha, J, \theta\right)$ such that $\alpha$ is $n_{1} \varphi_{1}-n_{2} \varphi_{2}$ and $J$ is a constant of the motion. This change of variables is accomplished by a generating function $S$, such as

$$
S=J_{\alpha}\left(n_{1} \varphi_{1}-n_{2} \varphi_{2}\right)+J\left(n_{2} \varphi_{1}+n_{1} \varphi_{2}\right) .
$$

Since $\alpha=\partial S / \partial J_{\alpha} \theta=\partial S / \partial J$ and $J_{i}=\partial S / \partial \varphi_{i}$, we have

$$
\begin{aligned}
\alpha & =n_{1} \varphi_{1}-n_{2} \varphi_{2}, & \theta & =n_{2} \varphi_{1}+n_{1} \varphi_{2} \\
J_{1} & =n_{1} J_{\alpha}+n_{2} J, & J_{2} & =n_{1} J-n_{2} J_{\alpha}
\end{aligned}
$$

For molecules, $H_{1}$ and $H_{2}$ are typically approximately quadratic functions of $J_{1}$ and $J_{2}$. In any event, in standard resonance theory $H-H_{\mathrm{M}}$ would be expanded as a quadratic function of $J_{\alpha}-J_{a}^{r} ; J_{\alpha}$, obtained in terms of $J_{1}^{\mathrm{r}}$ and $J_{2}^{\mathrm{r}}$ from eqn (7.6), is $\left(n_{1} J_{1}^{\mathrm{r}}-n_{2} J_{2}^{\mathrm{r}}\right) /\left(n_{1}^{2}+n_{2}^{2}\right)$. We now have from eqn (7.2)

$$
H=H_{1}\left(J_{1}^{\mathrm{r}}\right)+H_{2}\left(J_{2}^{\mathrm{r}}\right)-\frac{1}{2} \Omega\left(J_{\alpha}-J_{a}^{\mathrm{r}}\right)^{2}+A\left(J_{1}^{\mathrm{r}}, J_{2}^{\mathrm{r}}\right) \cos \alpha=\varepsilon
$$

where $\varepsilon$ is the energy of these two modes and

$$
-\Omega=n_{1}^{2} \partial^{2} H_{1} / \partial J_{1}^{2}+n_{2} \partial^{2} H_{2} / \partial J_{2}^{2}, \text { evaluated at }\left(J_{1}^{\mathrm{r}}, J_{2}^{\mathrm{r}}\right) .
$$

As a special case

$$
\Omega \cong \chi_{1}^{\circ} \omega_{1}^{\circ}+\chi_{2}^{\circ} \omega_{2}^{\circ} .
$$

Here $\chi_{1}^{\circ} \omega_{1}^{\circ}$ and $\chi_{2}^{\circ} \omega_{2}^{\circ}$ are the anharmonicity constants. Eqn (7.9) assumes the quadratic dependence of $H_{1}$ on $J_{1}$ to be good at all $J_{1}$ and to be independent of the energy excess of the other modes of the excited ligand. (They may contribute to an effective potential for mode 1.) Similar remarks apply to $\mathrm{H}_{2}$ and $J_{2}$.

We denote $H_{1}\left(J_{1}^{\mathrm{r}}\right)+H_{2}\left(J_{2}^{\mathrm{r}}\right)-\varepsilon$ by $B$. At the maximum of any curve in fig. 1 , $\frac{1}{2} \Omega\left(J_{\alpha}-J_{\alpha}^{r}\right)^{2}$ equals $B+A$, while on the separatrix one sees that $J_{\alpha}-J_{\alpha}^{\mathrm{r}}$ vanishes when $\alpha= \pm \pi$, i.e. when $B=A$. Thus the height of the maximum of the separatrix is $2 \sqrt{ }(A / \Omega)$ and the depth of the minimum is $-2 \sqrt{ }(A / \Omega)$.

Accordingly, if for any trajectory the maximum distance of $J_{\alpha}$ from $J_{\alpha}^{\mathrm{r}}$ satisfies

$$
\max \left|J_{\alpha}-J_{\alpha}^{\mathrm{r}}\right|<2 \sqrt{ }(A / \Omega)
$$

the projection of the trajectory on the $\left(J_{\alpha}, \alpha\right)$ plane in phase space will describe an "ellipse" in fig. 1. There is a "phase locking" (the $\varphi_{1}$ and $\varphi_{2}$ are seen to be correlated, because the range of $\alpha$ is now restricted), and $J_{\alpha}-J_{\alpha}^{\mathrm{r}}$ oscillates about zero. 
In terms of energy redistribution one sees from eqn (7.6) that it is extensive when $J_{\alpha}-J_{\alpha}^{\tau}$ oscillates about zero. For example, using eqn (7.10) we find at a given $J$

$$
\begin{aligned}
& \max \left|J_{1}-J_{1}^{\mathrm{r}}\right|<2 n_{1} \sqrt{ }(A / \Omega) \\
& \max \left|J_{2}-J_{2}^{\mathrm{r}}\right|<2 n_{2} \sqrt{ }(A / \Omega) .
\end{aligned}
$$

I.e. regardless of the initial values of $J_{1}$ and $J_{2}$, the modal actions $J_{1}$ and $J_{2}$ will oscillate about $J_{1}^{\mathrm{r}}$ and $J_{2}^{\mathrm{r}}$, as long as $J_{1}$ and $J_{2}$ lie in the resonance widths, eqn (7.11)-(7.12). If $J_{1}^{\mathrm{r}}$ and $J_{2}^{\mathrm{r}}$ are equal (as they are in intrinsic $1: 1$ near-resonant systems), there is an

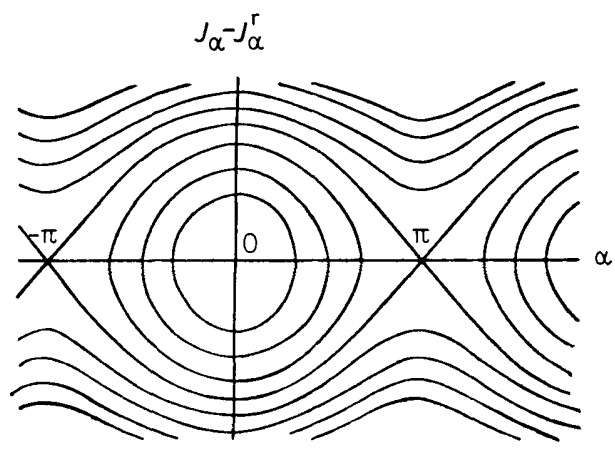

Fig. 1. Phase space plot of $J_{\alpha}-J_{\alpha}^{\mathrm{r}}$ against $\alpha$ for various values of $\varepsilon$ in eqn (7.7) and hence of the $B$ defined later in the text. The separatrix consists of the two curves passing through $\alpha= \pm \pi$. On the separatrix $B$ equals the $A$ in eqn (7.7).

equipartitioning. On the other hand if $\max \left|J_{1}-J_{1}^{\mathrm{r}}\right|$ lies outside the halfwidth $2 n_{1} \sqrt{ }(A / \Omega)$, there will be little or no tendency, classically, for such redistribution.

The time required for resonant transfer of energy between modes 1 and 2 is readily obtained from eqn (7.7). Well within the separatrix $\cos \alpha \operatorname{can}$ be expanded about $\alpha=$ 0 , yielding a harmonic-oscillator expression for the motion of $\alpha$. The period is then found to be $2 \pi / \sqrt{ }(A \Omega)$. The time for $J_{\alpha}-J_{\alpha}^{\mathrm{r}}$ to go from its maximum positive value to its maximum negative value is the half-period $\tau$,

$$
\tau=\pi / \sqrt{ }(A \Omega) \text {. }
$$

This is the typical time for the resonant intramolecular energy transfer when it is classically allowed. The conditions for the validity of resonance theory (e.g. the system must be sufficiently anharmonic) have been given by Chirikov [ref. (7), eqn (3.23)].

There may be other pairs of modes which involve motion of the bonds of $\mathrm{M}$, and which may be resonant. They can readily be included when they are isolated resonances. Currently we are obtaining information about the various resonances from power spectra of the bonds linking $M$ with the ligands, using power spectra of trajectories ${ }^{24}$ of the isolated ligands and of the molecule as a whole.

We turn next to quantum effects. Each classical resonance can embrace none, one or a number of pairs of resonant quantum states of modes 1 and 2. Extensive energy transfer among the two modes, and hence between the two ligands containing them, can occur quantum-mechanically between such pairs of resonant states even when it is classical forbidden, i.e. even when condition (7.10) is violated. The rate of transfer, reflected in the splitting of the quantum-mechanical levels of modes 1 and 2 , is then 
relatively low. This splitting can be calculated semiclassically ${ }^{21}$ based, in the present case, on eqn (7.7). The semiclassical results for the splitting in triatomic ABA molecules agreed with quantum-mechanical results very well when the splitting was large. ${ }^{21}$ However, when the splitting was small (corresponding to large values of $\left.\left|J_{\alpha}-J_{\alpha}^{\mathrm{r}}\right|\right)$ the error was considerable [e.g. the $|5,0\rangle \pm|0,5\rangle$ and $|4,0\rangle \pm|0,4\rangle$ cases in table II of ref. (21)]. In such cases a quantum-mechanical calculation would be needed. The error in these small-splitting cases is presumably due to replacing the $J_{1}$ and $J_{2}$ in $A\left(J_{1}, J_{2}\right)$ by constants. In such cases it might be better to use quantummechanical perturbation theory to calculate the splittings rather than a semiclassical formula [eqn. (7.7)].

\section{CONCLUSIONS AND SUMMARY}

The nature of anharmonic motion in molecules, classical and quantum, is discussed, and implications for a statistical theory (microcanonical) such as R.R.K.M., for unimolecular processes, are considered. An approximate statistical plus dynamical theory for intramolecular energy transfer between ligands across a central metal atom is given, with possible consequences for intramolecular laser-selective chemistry.

I should like to acknowledge the helpful collaboration (identified in the references) with D. W. Noid, M. L. Koszykowski, V. Lopez and T. Uzer on various portions of this research. The research was supported by a grant from the National Science Foundation. I am indebted to Drs. Vicente Lopez and David Wardlaw for their helpful comments. This is contribution no. 6775 .

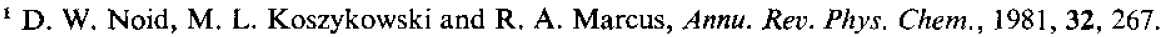

${ }^{2}$ P. Brumer, Adv. Chem. Phys., 1981, 47, 201.

${ }^{3}$ S. A. Rice, Adv. Chem. Phys., 1981, 47, 117.

4 M. Tabor, Adv. Chem. Phys., 1981, 46, 73.

${ }^{5}$ Nonlinear Dynamics, ed. R. G. Helleman, in Ann, N.Y. Acad. Sci., 1980, 357.

${ }^{6}$ N. C. Handy, Semiclassical Methods in Molecular Scattering and Spectroscopy, NATO Adv. Study Inst. Ser. C: Math. Physical Sci., ed. M. S. Child (Reidel, Dordrecht, 1980), p. 297.

${ }^{7}$ B. V. Chirikov, Phys. Rep., 1979, 52, 263.

${ }^{8}$ Topics in Nonlinear Dynamics. AIP Conf. Proc. No. 46, ed. S. Jorna, (Am. Inst. Phys., New York, 1978); Various articles of Am. Conf. Theor. Chem., in J. Phys. Chem., 1982, 86, $2113 \mathrm{ff}$.

${ }^{9} \mathrm{M}$. V. Berry, in ref. (8), p. 16.

${ }^{10}$ V. I. Arnol'd, Mathematical Methods of Classical Mechanics (Springer-Verlag, New York, 1978).

${ }^{11}$ I. C. Percival, Adv. Chem. Phys., 1977, 36, 1.

12 Stochastic Behavior in Classical and Quantum Hamiltonian Systems. Lecture Notes in Physics, Proc. Volta Mem. Conf., Como, Italy, ed. G. Casati and J. Ford (Springer-Verlag, New York, 1977), vol. 93.

13 J. Ford, Fundamental Problems in Statistical Mechanics, ed. E. G. D. Cohen (North Holland, Amsterdam, 1975), vol, 3, p. 215; J. Ford, Adv. Chem. Phys., 1973, 24, 155.

${ }_{14}$ M. V. Berry, Ann. Phys. N. Y., 1981, 131, 163.

${ }^{15}$ P. J. Richens and M. V. Berry, Physica, 1981, 2D, 495.

16 J. B. Keller, Ann, Phys., 1958, 4, 180.

${ }_{17}$ W. Eastes and R. A. Marcus, J. Chem. Phys., 1974, 61, 4301.

${ }^{18}$ D. W. Noid and R. A. Marcus, J. Chem. Phys., 1975, 62, 2119.

19 J. N. L. Connor, Chem. Phys. Lett., 1969, 4, 419.

${ }^{20}$ C. Jaffé and W. P. Reinhardt, J. Chem. Phys., 1982, 77, 5191.

${ }^{21}$ E. L. Sibert, J. T. Hynes and W. P. Reinhardt, J. Chem. Phys., 1982, 77, 3595.

22 V. Lopez and R. A. Marcus, to be published.

${ }^{23}$ T. Uzer, D. W. Noid and R. A. Marcus, to be published.

${ }^{24}$ M. L. Koszykowski, D. W. Noid and R. A. Marcus, J. Phys. Chem., 1982, 86, 2113 ; D. W. Noid, M. L. Koszykowski and R. A. Marcus, J. Chem. Phys., 1977, 67, 404.

${ }^{25}$ J. S. Hutchinson and R. E. Wyatt, Chem. Phys. Lett., 1980, 72, 378. 
${ }^{26}$ R. B. Gerber and R. A. Marcus, J. Chem. Phys, , to be submitted.

${ }^{27}$ R. B. Gerber, V. Buch and M. A. Ratner, Chem. Phys. Lett., 1982, 89, 171.

${ }^{28}$ R. A. Marcus, Ann. N.Y. Acad. Sci., 1980, 357, 169.

${ }^{29}$ K. G. Kay, J. Chem. Phys., 1980, 72, 5955.

${ }^{30}$ E. V. Shuryak, Sov. Phys. JETP, 1976, 44, 1070.

${ }^{31}$ D. W. Noid, M. L. Koszykowski, M. Tabor and R. A. Marcus, J. Chem. Phys., 1980, 72, 6169.

${ }^{32}$ M. D. Feit, J. A. Fleck Jr and A. Steiger, J. Comput. Phys., 1982, 47, 412.

${ }^{33}$ J. B. Delos and R. T. Swimm, Chem. Phys. Lett., 1977, 47, 76; R. T. Swimm and J. B. Delos, J. Chem. Phys., 1979, 71, 1706.

34 D. W. Noid and R. A. Marcus, J. Chem. Phys., 1977, 67, 559.

${ }^{35}$ D. W. Noid, M. L. Koszykowski and R. A. Marcus, J. Chem. Phys., 1979, 71, 2864.

${ }^{36}$ N. Saito, N. Ooyama, Y. Aizawa and H. Hirooka, Prog. Theor. Phys., Suppl., 1970, 45, 209.

${ }^{37}$ J. Ford, S. D. Stoddard and J. S. Turner, Prog. Theor. Phys., 1973, 50, 1547.

${ }^{38}$ V. Lopez and R. A. Marcus, Chem. Phys. Lett., 1982, 93, 232.

${ }^{39}$ R. T. Lawton and M. S. Child, Mol. Phys., 1979, 37, 1799.

${ }^{40}$ C. Jaffé and P. Brumer, J. Chem. Phys., 1980, 73, 5646.

${ }^{41} \mathrm{cf}$. D. W. Oxtoby and S. A. Rice, J. Chem. Phys., 1976, 65, 1676.

42 J. Jortner and R. D. Levine, Adv. Chem. Phys., 1981, 47, 1.

${ }^{43}$ R. A. Covaleskie, D. A. Dolson and C. S. Parmenter, J. Chem. Phys., 1980, 72, 5774.

44 J. E. Kenny, D. V. Brumbaugh and D. H. Levy, J. Chem. Phys. 1979, 71, 4757.

45 J. B. Hopkins, D. E. Powers and R. E. Smalley, J. Chem. Phys., 1980, 73, 683.

${ }^{46}$ P. Rogers, D. C. Montague, J. P. Frank, S. C. Tyler and F. S. Rowland, Chem. Phys. Lett., 1982, 89, 9 . 